\title{
Within-colony spatial segregation leads to foraging behaviour variation in a seabird
}

\author{
Sonia Sánchez ${ }^{1, *}$, Richard D. Reina ${ }^{1}$, Akiko Kato ${ }^{2}$, Yan Ropert-Coudert ${ }^{2}$, \\ Catherine Cavallo ${ }^{1}$, Graeme C. Hays ${ }^{3}$, André Chiaradia ${ }^{4}$ \\ ${ }^{1}$ School of Biological Sciences, Monash University, Clayton, Victoria 3800, Australia \\ ${ }^{2}$ Centre d'Etudes Biologiques de Chizé, CNRS - Université de La Rochelle UMR 7372, 79360 Villiers-en-Bois, France \\ ${ }^{3}$ Deakin University, Geelong, Victoria 3280, Australia \\ ${ }^{4}$ Research Department, Phillip Island Nature Parks, PO Box 97, Cowes, Victoria 3922, Australia
}

\begin{abstract}
Central-place foraging in large seabird colonies leads to high levels of intra-specific competition for food resources, often resulting in between-colony spatial segregation. However, little is known about within-colony variation in foraging behaviour that may arise from breeding locations. Using little penguins Eudyptula minor from a large colony (ca. 32000 individuals) on Phillip Island (Australia), we present a novel approach combining GPS, diving, acceleration and bathymetry data. We investigated within-colony variation in 3-dimensional distribution of prey encounters and its consequences for foraging behaviour and breeding success. Over 1 breeding season, we simultaneously tracked 63 little penguins from 2 breeding sites located $\sim 2 \mathrm{~km}$ apart and monitored their breeding success. We recorded 58452 dives, of which 11992 had prey encounter events associated. Results revealed strong spatial foraging segregation between sites throughout the breeding season and differences between sites in prey encounter depth during chick-rearing (mean $\pm \mathrm{SE}, 11.8 \pm 0.2 \mathrm{~m}$ vs. $17.3 \pm 0.3 \mathrm{~m}$ ). Birds from one site foraged in deeper waters and apparently experienced higher levels of competition, resulting in lower prey encounter rates and lower foraging efficiency (i.e. body mass gain after foraging trips), hence these birds seemed less successful. However, breeding success was high ( $>1.5$ chicks fledged per pair) at both sites, indicating that food was not a limiting factor that year. Nonetheless, breeding success records over the last $12 \mathrm{yr}$ showed that the site where birds foraged at deeper depths produced fewer chicks. Our findings highlight the importance of understanding small-scale spatial segregation to capture foraging behaviour variation within large seabird colonies.
\end{abstract}

KEY WORDS: Prey encounter - Diving behaviour - Colonial breeding - Foraging efficiency · Breeding ecology $\cdot$ Bio-logging $\cdot$ Acceleration $\cdot$ Eudyptula minor

\section{INTRODUCTION}

Colonial breeding, when a number of individuals breed within a central place from which they repeatedly depart in search of food (Wittenberger \& Hunt 1985), provides many benefits to diverse taxonomic groups, including reduced predation pressure (Robinson 1985, Danchin \& Wagner 1997), exchange of social information (Dall et al. 2005, Robinson et al. 2009, Evans et al. 2016) and increased opportunities for finding a high-quality mate (Dubois et al. 1998).

${ }^{*}$ Corresponding author: soniasg9@gmail.com
However, individuals also pay fitness costs, such as transmission of parasites and diseases (Møller 1987, Danchin \& Wagner 1997), competition for food resources (Furness \& Birkhead 1984, Forero et al. 2002) and competition for nest space (Ramos et al. 1997). This trade-off is especially widespread among seabirds, of which $98 \%$ of species nest in colonies that can vary from aggregations of few individuals to over 1 million birds (Wittenberger \& Hunt 1985).

Seabirds are central-place foragers while breeding, as they must frequently return to the colony to in-

(C) The authors 2018. Open Access under Creative Commons by Attribution Licence. Use, distribution and reproduction are unrestricted. Authors and original publication must be credited. 
cubate their eggs or to feed their offspring (Rolland et al. 1998). During brood provisioning, breeding adults need to maintain their body condition while meeting the increasing energetic requirements of their young (Gales \& Green 1990, Bevan et al. 2002, Ratcliffe et al. 2018). In order to balance this situation, some seabirds alternate between short trips to feed their offspring and long trips to restore and maintain their own body condition (Weimerskirch et al. 1997, Ropert-Coudert et al. 2004, Saraux et al. 2011), increase foraging effort to meet chick growth requirements (Ratcliffe et al. 2018), switch diet to more energy-dense prey (Booth \& McQuaid 2013, Handley et al. 2017) and modify diving behaviour (Booth et al. 2018).

Competition for food resources has been proposed as a regulating mechanism of pelagic seabird populations by prey depletion near the colony ('Ashmole's halo'), forcing individuals to forage further (Storer 1952, Ashmole 1963). Cairns (1989) argued that Ashmole's halo did not consider interactions between neighbouring colonies and, alternatively, predicted complete spatial segregation between adjacent colonies (hinterland model). There is evidence that supports both models (e.g. Lewis et al. 2001, Ainley et al. 2003, Grémillet et al. 2004, Elliott et al. 2009). More recently, Wakefield et al. (2013) found that neighbouring colonies of a wide-ranging seabird, the northern gannet Morus bassanus, forage in largely mutually exclusive areas determined by densitydependent competition. The model developed by Wakefield et al. (2013) suggested that spatial segregation could be enhanced by information transfer among individuals at sea and at the colony. In fact, the use of social information can facilitate group foraging (Weimerskirch et al. 2010, Sutton et al. 2017) and optimise foraging efficiency (McInnes et al. 2017) in some seabirds. Hence, indirect competition for food resources and social behaviour could be mechanisms simultaneously underlying spatial foraging segregation.

Most studies investigating foraging segregation between neighbouring colonies are based on wideranging seabirds from colonies separated by tens or hundreds of kilometres. Studies at a much finer spatial scale, i.e. within the same colony, using shortranging seabirds are scarce and often limited to chick-rearing stages. Spatial foraging segregation between colony members can arise from intrinsic individual factors such as sex and age (e.g. Weimerskirch et al. 2006, Pelletier et al. 2014, Cleasby et al. 2015). Often, bird colonies are not defined entities (Jovani et al. 2008) and can be divided into several discrete and isolated sub-colonies or breeding sites
(Waggitt et al. 2014), each of which may promote specific foraging behaviours (Masello et al. 2010, Ceia et al. 2015) and differences in breeding success (Hipfner et al. 2007). Yet, such within-colony asymmetries associated with breeding locations have received little attention, although they could have important implications for conservation policies and for individuals' fitness and life history, especially in species with high nest-site fidelity.

Little penguins Eudyptula minor are the smallest of all penguin species and have one of shortest foraging ranges (around $20 \mathrm{~km}$ ) among seabirds during the breeding season (Collins et al. 1999, Preston et al. 2008). Parents alternate nest attendance duties and foraging during incubation and guard (i.e. when chicks are between 1 and 19 d old) reproductive stages, with incubation trips lasting 1-9 d (Kato et al. 2008) and guard trips always lasting $1 \mathrm{~d}$ (Chiaradia \& Kerry 1999, Preston et al. 2010). During the postguard period, parents only return at night to feed the young (Chiaradia \& Kerry 1999) and alternate between short and long trips (Saraux et al. 2011). Phillip Island (south-eastern Australia) is one of the largest little penguin colonies in the world, a mega-colony by little penguin standards, with an estimated population of 28000-32000 individuals (Sutherland \& Dann 2014), hence high levels of intra-specific competition are expected. Burrows are located on the coastline of the $3 \mathrm{~km}$ long Summerland peninsula (Dann 1991) and penguins come ashore at dusk and leave at sunrise. Penguins come ashore and leave using established pathways, delimited by rocky outcrops, providing a natural division of the whole colony into sub-colonies (hereafter termed 'sites'). This division results in a good model to investigate whether such spatial structure leads to foraging behaviour and breeding success asymmetries within the colony.

Here, we studied the 3-dimensional foraging behaviour and the breeding success of little penguins at 2 neighbouring sites located $2 \mathrm{~km}$ apart within the Phillip Island colony. We investigated if penguins from each site shared the same foraging area or segregated in space and compared the bathymetry of their used areas, as it can influence their diving behaviour (Chiaradia et al. 2007, Meyer et al. 2017). We hypothesised that (1) there is a spatial foraging segregation between sites, following the models of Cairns (1989) and Wakefield et al. (2013), (2) there are differences in the foraging activity and efficiency between sites, and (3) breeding success differs between sites as an ultimate consequence of the differences in foraging behaviour. 


\section{MATERIALS AND METHODS}

\section{Study site and experimental design}

Field work was conducted simultaneously at 2 neighbouring little penguin breeding sites, Penguin Parade ${ }^{\circledR}$ and Radio-tracking Bay, located $2 \mathrm{~km}$ apart, on Phillip Island, Victoria, Australia $\left(38^{\circ} 31^{\prime} \mathrm{S}, 145^{\circ}\right.$ 09' E). Penguin Parade is situated on the eastern margin of the colony and contains both artificial nest boxes and natural burrows, while Radio-tracking Bay is located west of Penguin Parade, sharing its eastern and western margins with other sites, and contains only natural burrows (Fig. 1). We simultaneously monitored foraging behaviour and breeding performance in a subset of 100 nests at each site.

We tested if there were site-specific patterns in the 3-dimensional habitat use of birds by combining horizontal and vertical tracking with acceleration data collected during the 2015/2016 breeding season. To do so, we measured the spatial overlap between sites in the horizontal distribution and depth of prey encounters over the 3 breeding stages: incubation, guard and post-guard. We then compared the bathymetry between sites within the foraging area used at each stage. In order to investigate the consequences of these 3-dimensional patterns, we compared the foraging activity (i.e. number of dives with at least 1 prey encounter event per hour of diving on each foraging trip) and efficiency (i.e. adult body mass change after foraging trips) between sites. We then determined the breeding performance of each site by calculating the peak chick mass (Chiaradia \& Nisbet 2006) and breeding success as the number of chicks fledged per pair.

\section{Bird instrumentation and tracking}

Between September and November of 2015, we tracked 74 little penguins from Penguin Parade and Radio-Tracking Bay using GPS and accelerometer data loggers. We recovered data from 71 birds, but

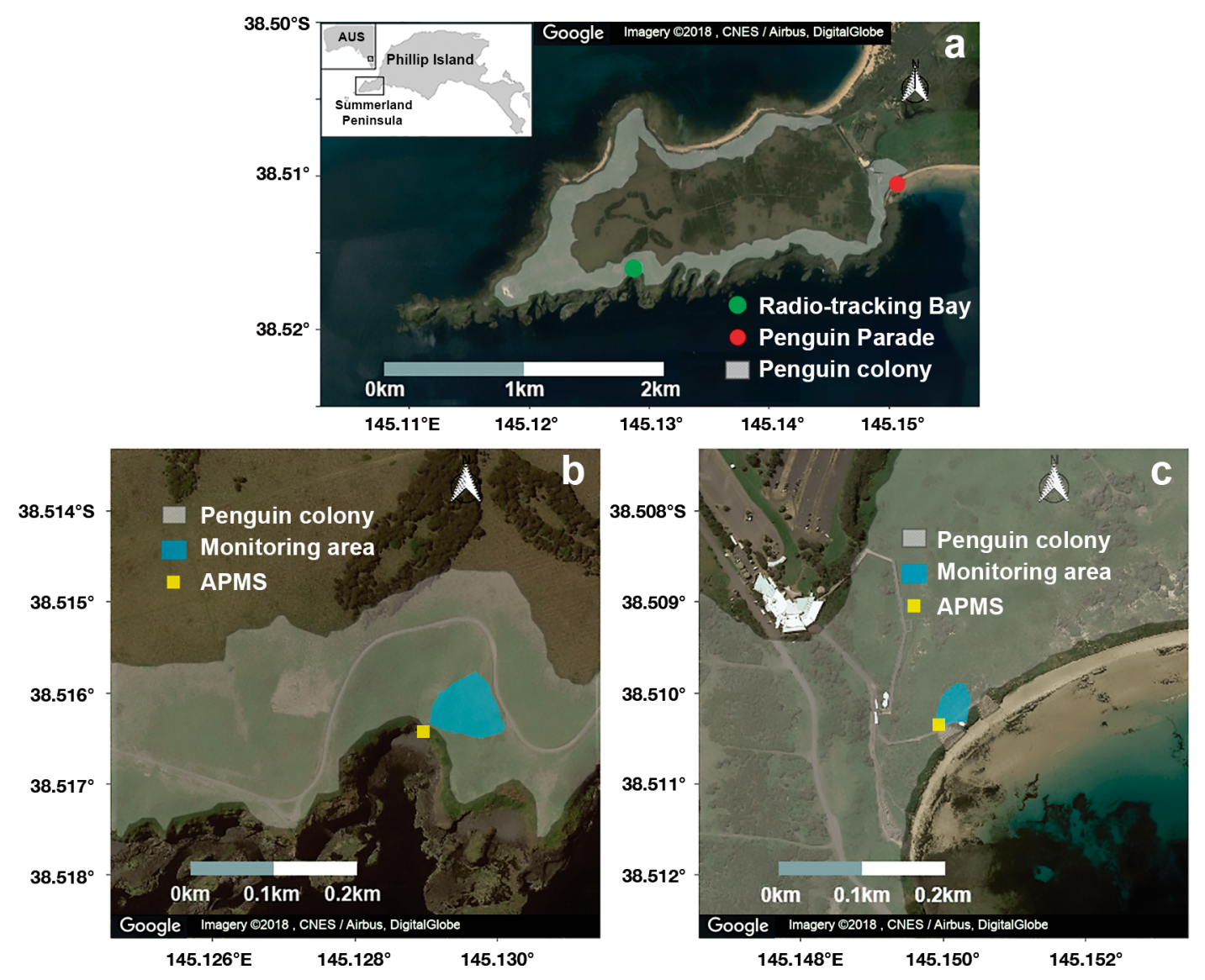

Fig. 1. Satellite images showing the geography of the study area on the Summerland Peninsula, Phillip Island, Victoria, Australia. (a) Distribution of the little penguin Eudyptula minor colony on Phillip Island adapted from Sutherland \& Dann (2014) and location of the breeding sites monitored in this study. Also shown are the areas containing the monitored burrows (blue) and location of the automated penguin monitoring system (APMS) at each breeding site: (b) Radio-tracking Bay and (c) Penguin Parade 
only complete trips with data from both GPS and accelerometer were considered for further analysis. In total, 63 foraging trips over incubation $(\mathrm{n}=19)$, guard ( $\mathrm{n}=27$; chicks between 1 and $19 \mathrm{~d}$ old) and post-guard ( $\mathrm{n}=17$; chicks are unattended during the daytime in the colony) were analysed. For each breeding stage, birds from both sites were tracked simultaneously, aiming to maintain the same sample size per site and sex.

Adult birds were captured in their nest boxes or natural burrows and weighed $( \pm 10 \mathrm{~g})$ using a spring balance. A GPS (modified as per below; CatTrack 1, 380 mA lithium-ion battery, Catnip Technologies) and a tri-axial accelerometer data logger were attached with Tesa ${ }^{\circledR}$ tape (Beiersdorf) to each bird's middle and lower back, respectively, as per Preston et al. (2008). All accelerometers also had temperature and pressure sensors, and models used were either AXY-Depth $(31 \times 12 \times 11 \mathrm{~mm}$; Technosmart $)$, ORI400D3GT (45 × $12 \mathrm{~mm}$; Little Leonardo) or Wacu $(21 \times 13$ $\times 4 \mathrm{~mm}$; IPHC-DEPE). The original packaging of the GPS loggers was removed and the devices were waterproofed inside a heat-shrink tube. Dimensions of customised units were approximately $50 \times 20 \times$ $8 \mathrm{~mm}$. The 2 devices together (GPS and accelerometer) weighed $\sim 35 \mathrm{~g}$ in air, and the minimum and maximum percentage of body mass for any logger deployment was 2.4 and $4.2 \%$, respectively. The cross-section area of the loggers was $\sim 3 \%$ of the bird's frontal area (as calculated by Ropert-Coudert et al. 2007). Birds were immediately returned to their nests after the device attachment and were recaptured in their nests after a single foraging trip to retrieve the loggers. Total handling time was less than 5 min, and the integrity of the plumage was preserved during deployments and retrievals.

GPS loggers recorded at-sea positions from 04:00 to $21: 00 \mathrm{~h}$ every $120 \mathrm{~s}$ for incubation and postguard trips and every $20 \mathrm{~s}$ for guard trips. Accelerometers recorded depth (ORI400-D3GT, range 0-300 $\mathrm{m}, \pm 0.1 \mathrm{~m}$ ) or pressure (AXY-Depth and Wacu, range $0-30$ bar, \pm 0.5 mbar) and temperature (range -10 to $50^{\circ} \mathrm{C}, \pm 0.1^{\circ} \mathrm{C}$ ) every second, as well as acceleration along the 3 body axes of the penguins: longitudinal (surge), dorso-ventral (heave) and lateral (sway), between -20 and $20 \mathrm{~m} \mathrm{~s}^{-2}$ at $25 \mathrm{~Hz}$ for incubation and post-guard trips and $50 \mathrm{~Hz}$ for guard trips. The sampling intervals for the different breeding stages were selected as a compromise between the logger battery life and variability in foraging trip duration during incubation and post-guard (Chiaradia \& Kerry 1999, Kato et al. 2008, Saraux et al. 2011).

\section{Depth, acceleration and GPS data processing}

Dive analysis was conducted on depth and acceleration data downloaded from the accelerometer data loggers and using a purpose-written software in Igor Pro Version 6.37 (Wavemetrics; see Kato et al. 2006). For AXY-Depth and Wacu accelerometers, pressure in millibars was converted to water depth $(\mathrm{m})$. The software considered a dive started when birds descended from the water surface and ended when they returned to it. Dives were analysed sequentially for each foraging trip. According to the resolution of the loggers and waves at the surface (Kato et al. 2006), only dives $>1 \mathrm{~m}$ were considered for the analysis. Each dive was divided in 3 phases: descent, bottom and ascent phases. The start and end of the bottom phase were defined as the first and last time in a dive when the absolute rate of change of depth was lower than $0.25 \mathrm{~m} \mathrm{~s}^{-1}$ (Ropert-Coudert et al. 2006). The times before and after the bottom phase within the dive were considered the descent and ascent phases, respectively. The deepest point reached during a dive was termed maximum depth.

Prey encounter events were identified using acceleration data. Wingbeats are apparent in acceleration signals as a series of regularly alternating increases and decreases of the heave and surge axes (Watanuki et al. 2006). During prey pursuit, wingbeat signals suddenly increase in frequency and amplitude, resulting in a peak in the overall acceleration (RopertCoudert et al. 2006, Zimmer et al. 2011a). The vectorial dynamic body acceleration (VDBA) is the vectorial sum of the 3 axes' dynamic acceleration, being a proxy of the whole body activity (Gleiss et al. 2011):

$$
\operatorname{VDBA}=\sqrt{\left(A_{x}^{2}+A_{y}^{2}+A_{z}^{2}\right)}
$$

where $A_{x}, A_{y}$ and $A_{z}$ are the dynamic acceleration of surge, heave and sway axes, respectively. The dynamic acceleration of each axis was obtained by subtracting the static acceleration (i.e. total acceleration of each axis smoothed over $1 \mathrm{~s}$ ) from the total acceleration (Shepard et al. 2008). Finally, VDBA values were averaged every second for further analysis. Evaluating the frequency of VDBA during the 3 dive phases, we found an upper inflection point at $0.5 \times g$ common to all birds (see Fig. S1 in Supplement 1 at www.int-res.com/articles/suppl/m606p215_supp1.pdf). We used this upper inflection point as a threshold to detect prey encounter events. When the VDBA became higher than $0.5 \times g$ within a dive, we considered the penguin had encountered and pursued prey, resulting in a peak in the overall acceleration (see Fig. 2 for an illustrative example). The first $5 \mathrm{~m}$ of descent 


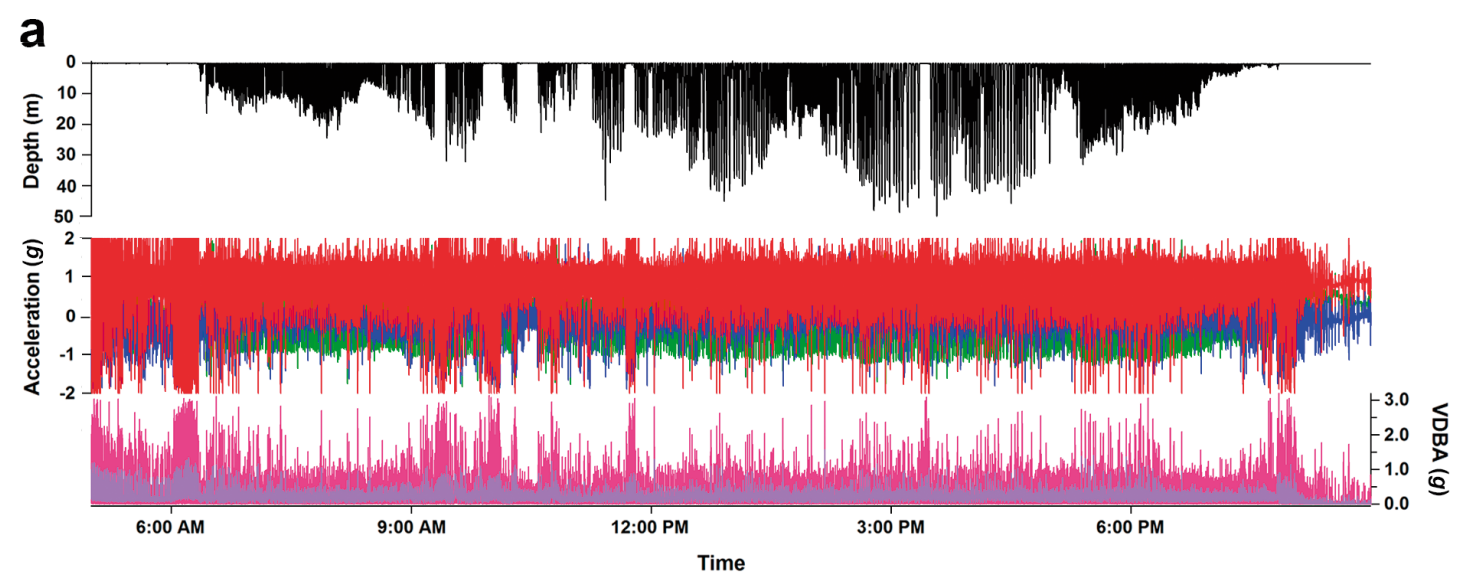

b
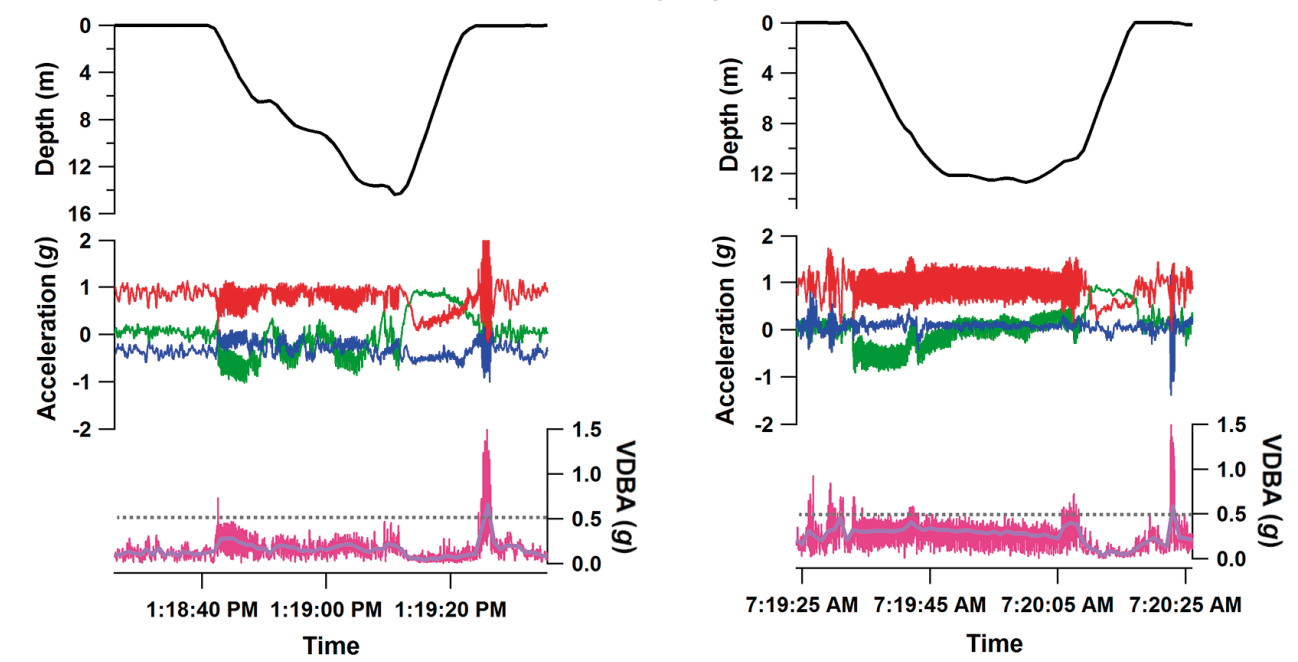

Dives with prey encounter
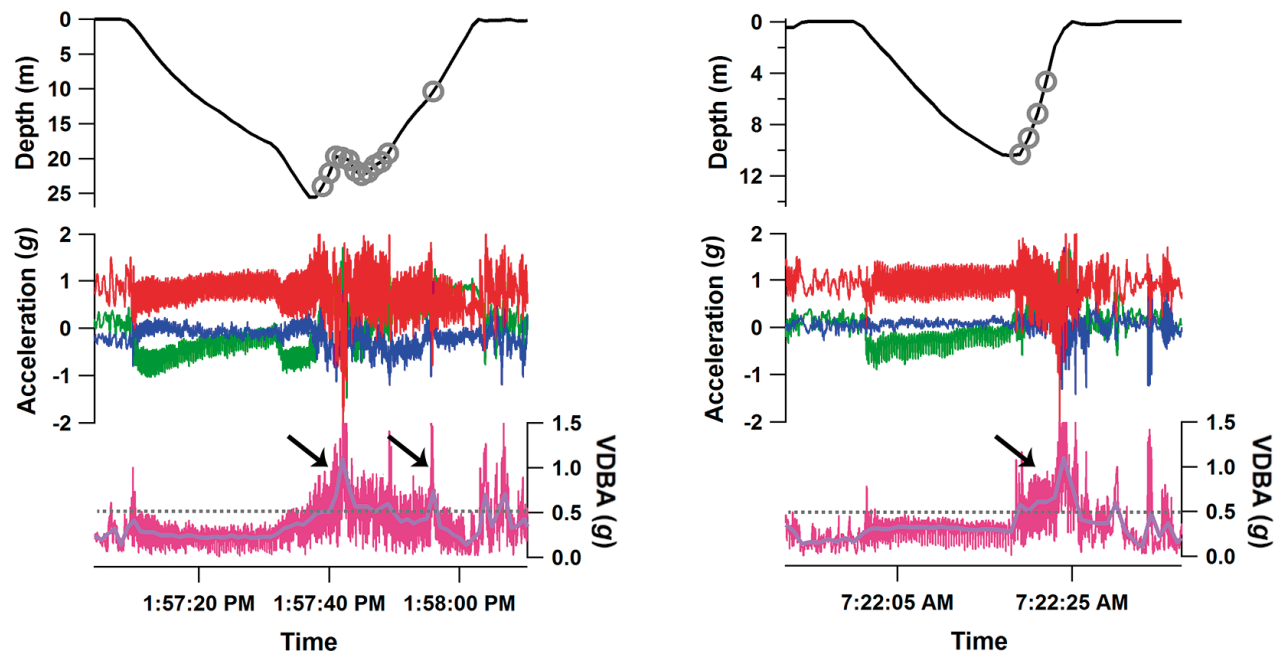

Fig. 2. (a) Depth and acceleration profiles for a complete foraging trip by a little penguin during the guard stage. Diving activity (top), acceleration at $50 \mathrm{~Hz}$ (middle) on the surging (green), heaving (red) and swaying (blue) axes and vectorial dynamic body acceleration (VDBA; bottom) at $50 \mathrm{~Hz}$ (pink) and smoothed over $1 \mathrm{~s}$ (purple). (b) Examples of dives without and with a prey encounter event. Each prey encounter event (1 s) is indicated by the grey circles on the diving profiles and the black arrows on the VDBA graph. Acceleration at $50 \mathrm{~Hz}$ is shown on the surging (green), heaving (red) and swaying (blue) axes. VDBA at $50 \mathrm{~Hz}$ (pink) and smoothed VDBA (purple). The grey dotted line on the VDBA graph indicates the prey encounter and pursuit threshold 
phases were excluded from VDBA threshold detection because birds beat their wings vigorously to overcome buoyancy at the beginning of a dive (Zimmer et al. $2011 a, b)$. Bottom and ascent phases from all dives, regardless of their depth, were kept for prey encounter detection purposes. For each dive with a prey encounter, the depth at which the first VDBA point exceeded $0.5 \times g$ was considered as the prey encounter depth $(\mathrm{m})$. For each trip, the foraging activity was measured by calculating a prey encounter rate as the number of dives with prey encounters divided by the sum of all dive durations $\left(\mathrm{h}_{\text {div }}\right)$.

The distance between consecutive GPS locations was calculated using the Haversine algorithm from the 'geosphere' package (Hijmans 2016) within the R statistical environment in order to estimate the swimming speed between points. We excluded GPS locations at the colony and locations with a swimming speed of $0 \mathrm{~m} \mathrm{~s}^{-1}$ (i.e. duplicated points) or greater than $3.3 \mathrm{~m} \mathrm{~s}^{-1}$ (i.e. maximum swimming speed for little penguins; Preston et al. 2008) from the dataset, as they were considered either erroneous locations or did not belong to the foraging phase of the trips. We allocated a location to each dive with a prey encounter by linearly correlating the timestamp at the beginning of each dive event with the closest GPS locations recorded before and after the dive. In some instances, the foraging trip exceeded (by a maximum of $2 \mathrm{~h}$ ) the battery life of the GPS logger, and the location of dives recorded after the GPS device stopped logging positions could not be determined. However, we included these trips in the analysis, as dives without a location only accounted for $4 \%$ of the total dives with prey encounter, indicating that at the end of the trip, birds were mainly travelling back to the colony without actively foraging. In order to assess the accuracy of prey encounter locations, we counted the number of dives with prey encounters that occurred during gaps in the GPS data that exceeded $5 \mathrm{~min}$. As this corresponded to only $3 \%$ of the prey encounter dives, we kept these prey encounter locations in the spatial analysis.

\section{Automated penguin monitoring system}

At each site, adult birds attending the monitored nests were identified with passive identification transponders (Allflex and Trovan). Two automated penguin monitoring systems (APMS), 1 at each site, located on the main penguin pathway, recorded the transponder number, date/time and individual body mass of adult birds leaving at sunrise and returning ashore at dusk (Kerry et al. 1993, Chiaradia \& Kerry 1999). We only considered APMS data recorded during the tracking period and from known breeding adults attending the monitored burrows. In order to eliminate potential erroneous adult mass, we filtered out adult mass lower than the 5th percentile $(712 \mathrm{~g})$ and higher than the 95th percentile (1547 g). Two different adult body masses were examined for a given foraging trip: 'departure mass', which was the mass of the bird leaving the colony at sunrise, and the 'return mass', which was the mass of the bird returning to the colony at sunset. These 2 body masses were considered to belong to the same foraging trip when their records were consecutive in date and time for a given transponder number and the trip duration was not longer than $1 \mathrm{~d}$ in guard and $21 \mathrm{~d}$ in incubation and post-guard. The difference between the 'return mass' and the 'departure mass' was used as an estimate of the mass (in $\mathrm{g}$ ) of food obtained over the foraging trips, i.e. foraging efficiency (Saraux et al. 2011).

\section{Reproductive performance}

During the 2015/2016 breeding season, we monitored 100 penguin nests at each site, 3 times $w^{-1}$ at Penguin Parade and once weekly at Radio-tracking Bay, to assess laying date, hatching date and fledging success. We identified adults in the nests by scanning their transponder numbers. New birds were injected with transponders as per Chiaradia \& Kerry (1999), and sex was determined through measurements of bill morphometrics with a $91 \%$ accuracy (Arnould et al. 2004). During each nest visit, chicks were weighed $( \pm 10 \mathrm{~g})$, using a spring balance, from hatching until fledging (Saraux et al. 2011). We determined the peak chick mass for each fledged chick (Chiaradia \& Nisbet 2006) during the first clutch of the season, i.e. when breeding adults were tracked. Due to the monitoring frequency, this was the most comparable chick parameter between sites.

In order to assess the overall success, breeding success was determined at each site as the number of chicks fledged per breeding pair (Chiaradia \& Kerry 1999, Chiaradia \& Nisbet 2006) over 12 yr, from 2004 until 2015, following the same monitoring protocol as for the 2015/2016 season.

\section{Data analysis}

All statistical analyses were conducted using $\mathrm{R}$ 3.4.3 (R Core Team 2017). 
Three-dimensional spatial pattern of prey encounter

Geographic coordinates of prey encounter events were projected into a Universal Transverse Mercator projection (zone 55S, WGS 84 Datum). For each stage and site, we used the prey encounter locations to estimate the homerange (95\% utilisation distribution, UD) and core range (50 and $25 \%$ UD) of prey pursuits using kernel density analysis and estimated the areas of each contour with the $\mathrm{R}$ package 'adehabitatHR' (Calenge 2006). Following Lascelles et al. (2016), the smoothing factor used in the kernel density analysis was calculated as the average area-restricted search (ARS) behaviour exhibited across all trips within each breeding stage. The ARS was assessed by first passage time analysis with the fpt function of the R package 'adehabitatLT' (Calenge 2006). The size of the grid for the kernel density analysis was set at $400 \mathrm{~m}$ as a compromise between the resolution of the bathymetry data obtained from Geoscience Australia (250 m × $250 \mathrm{~m}$ resolution) and the computation time. We also estimated the 95 and $25 \%$ contours of prey pursuits for each individual trip following the above procedure and extracted the median bathymetry within each contour.

The extent of within-stage overlap between Penguin Parade and Radio-tracking Bay home and core ranges was estimated using the UD overlap index (UDOI; Fieberg \& Kochanny 2005) with the kerneloverlaphr function of the R package 'adehabitatHR'. Using UDOI as our metric, we then used a randomisation procedure (see customised R function in Supplement 2 at www.int-res.com/articles/suppl/m606p215_ supp2.txt) to test the null hypothesis that there was no difference in the spatial distribution of prey encounter events between sites within each breeding stage (Breed et al. 2006, Cleasby et al. 2015). For each stage, site was randomly assigned to tracks (i.e. prey encounter locations) using the same site/sex ratio as the observation, and kernel analysis and overlap (i.e. UDOI) estimation were applied. This procedure was iterated 1000 times for the 95, 50 and 25\% contours of each breeding stage. The empirical p-value (North et al. 2002) was determined as the proportion of random overlaps that were smaller than the observed overlap (i.e. the probability that spatial segregation was driven by chance). When the empirical p-value was $<0.05$, we considered the observed overlap to be significantly lower than expected by chance.

We compared the bathymetry of the foraging areas between sites for each breeding stage by performing a permutation test using Brunner and Munzel's generalised Wilcoxon test (Neubert \& Brunner 2007, Ruxton \& Neuhäuser 2013). The null hypothesis tested was that there were no differences in the bathymetry use between sites. For the 95 and $25 \%$ contours of each breeding stage, we first estimated the generalised Wilcoxon test statistic between sites of the observed median bathymetry of individual trips. We then ran 10000 permutations where site was randomly assigned to the median bathymetry of each individual trip, maintaining the sample size for each site, and the generalised Wilcoxon test statistic was estimated for each permutation. The empirical pvalue was determined as the proportion of random absolute test statistics larger or equal to the absolute observed test statistic. Empirical p-values $<0.05$ were considered to show significant differences between sites in the bathymetry within the foraging area used.

The influence of site on prey encounter depth was modelled separately for each breeding stage using linear mixed effects models (LMMs) from the R package 'nlme' (Pinheiro et al. 2016). Site and sex were included as fixed effects and bird ID was included as a random intercept to avoid pseudo-replication and account for any individual differences in dive behaviour of birds. A correlation structure across bird ID was incorporated in the model using the first-order autoregressive to allow for similarities between dives that occurred in temporal clusters. For each LMM, model selection of the fixed effects was conducted by maximum likelihood (ML) estimation, inspection of residual plots and the use of Akaike's information criterion (AIC). The most appropriate random structure was then determined via restricted maximum-likelihood (REML) estimation and selected by a comparison of AIC and residual plots (Zuur et al. 2009). The most parsimonious model was then refitted using REML to obtain parameter estimates and associated p-values (Zuur et al. 2009).

\section{Foraging activity}

The influence of site on the prey encounter rate of each foraging trip was modelled separately for each breeding stage using a generalised least square (GLS) approach from the R package 'nlme' (Pinheiro et al. 2016). Site and sex were included as fixed effects. Trip duration was also included for incubation and postguard models to account for trip duration flexibility in these stages (Kato et al. 2008, Saraux et al. 2011). We detected heterogeneity of variances between sites, which was incorporated in the model via the weights argument in the gls function. Model selection was conducted using AIC, and the final model was validated inspecting residual plots (Zuur et al. 2009). 
Adult mass change and reproductive performance

Differences between sites in the mass change after foraging trips and in the adult body mass at the beginning of the trips were compared using LMMs from the R package 'nlme' (Pinheiro et al. 2016) with bird ID as a random intercept. Each breeding stage was modelled separately, and trip length was incorporated as a fixed effect in the incubation and postguard models. Model selection and validation were conducted following the same procedure as for the LMMs described above.

Differences in the reproductive performance of the first clutch of the 2015/2016 season between sites were assessed by comparing peak chick mass between sites fitting a linear model.

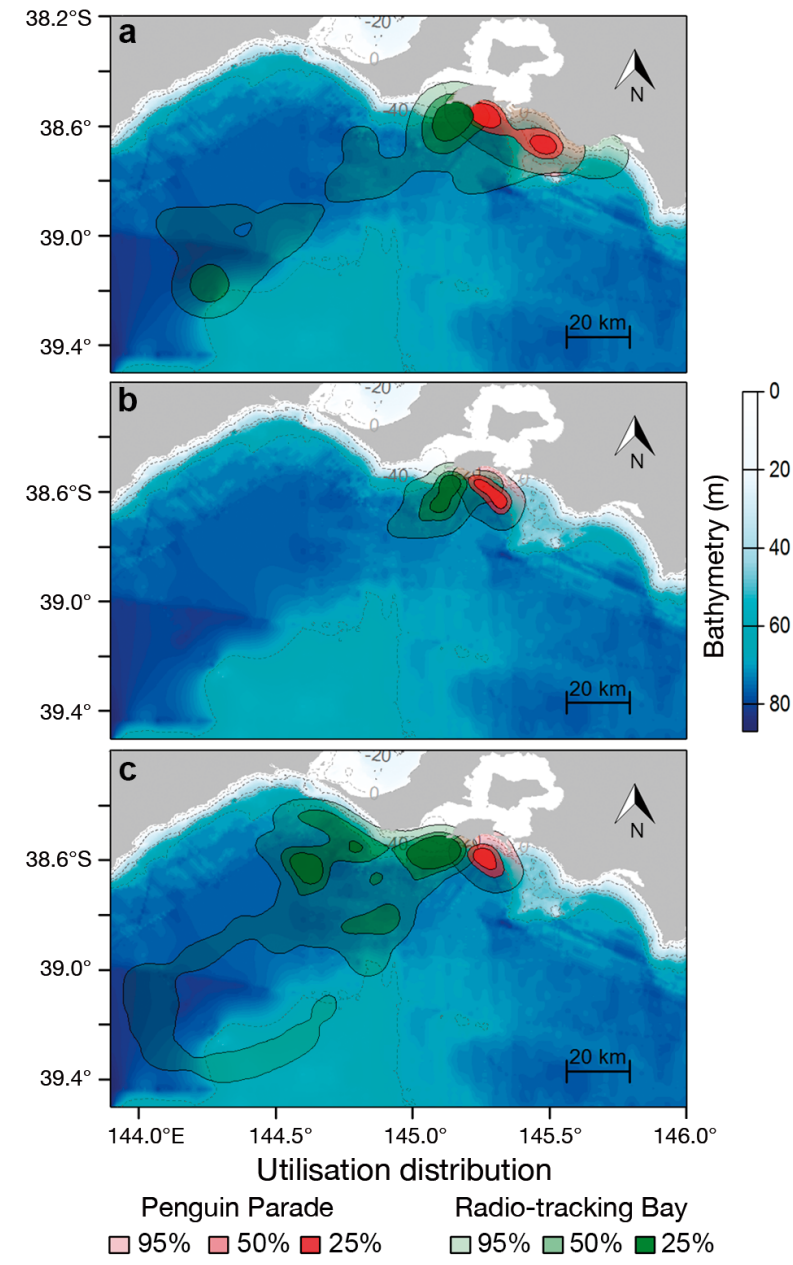

Fig. 3. Kernel density utilisation distribution of prey encounter locations during (a) incubation, (b) guard and (c) post-guard stages of little penguins at Penguin Parade (red) and Radiotracking Bay (green). Grid size is $400 \mathrm{~m}$, smoothing factor is $4.4 \mathrm{~km}$ for incubation, $3.7 \mathrm{~km}$ for guard and $2.96 \mathrm{~km}$ for postguard. Smoothing factors were calculated for each stage following Lascelles et al. (2016)

\section{RESULTS}

\section{Three-dimensional spatial patterns of prey encounter}

Tracking data consisted of 63 complete foraging trips by 63 little penguins over 1 breeding season (Table S1 in Supplement 1). The total number of dives made across all foraging trips at both sites was 58452 (Penguin Parade: 27686 dives; Radio-tracking Bay: 30766$)$, of which 11992 dives had prey encounter events associated (Penguin Parade: 5241 dives; Radio-tracking Bay: 6751 dives). We were not able to allocate a location for 2487 dives (527 with prey encounter), and therefore they were not included in the spatial analysis.

Birds from Penguin Parade mainly foraged southeast of their breeding site, while birds from Radiotracking Bay foraged south-west of their site (Fig. 3). Consequently, and based on the randomisation tests of prey encounter spatial overlap, we found that little penguins presented a significant spatial foraging segregation between sites for all breeding stages, except for the incubation home-range (30\% overlap, $\mathrm{p}=0.06$, Table 1, Fig. 3). There was no overlap $(\mathrm{UDOI}=0)$ in the core areas of prey encounters between sites during guard and post-guard (Table 1, Fig. 3). Birds from Radio-tracking Bay consistently used larger areas over the breeding season than birds from Penguin Parade (Fig. 3, Table A1 in the Appendix), with the maximum differences observed during incubation (50\% core area: 519 vs. $296 \mathrm{~km}^{2}$, respectively; Table A1) and post-guard (50\% core area: 1004 vs. $109 \mathrm{~km}^{2}$, respectively; Table A1).

Table 1. Estimated utilisation distribution (UD) overlap index (UDOI) in UDs between Parade and Radio-tracking Bay little penguins within incubation, guard and post-guard breeding stages. Empirical p-value (p): the proportion of 1000 randomised overlaps that were smaller than the observed overlap. Randomised overlaps were only calculated if observed overlap was $>0$

\begin{tabular}{|cccc|}
\hline \multirow{2}{*}{ UD (\%) } & Stage & UDOI & $\mathrm{p}$ \\
\hline 95 & Incubation & 0.3 & 0.06 \\
& Guard & 0.14 & 0.001 \\
& Post-guard & 0.01 & 0.01 \\
& Incubation & 0.007 & 0.001 \\
& Guard & 0 & - \\
& Post-guard & 0 & - \\
& Incubation & 0 & - \\
& Guard & 0 & - \\
& Post-guard & 0 & \\
& & & \\
& & &
\end{tabular}


Single long foraging trips occurred in the incubation and post-guard stages with individuals from Radiotracking Bay, but were rare, occurring in 1 of 9 and 1 of 7 foraging trips, respectively. These long trips resulted in the home-range foraging areas extending further than $100 \mathrm{~km}$ to the SW (Fig. 3a,c). However, the finding of a significant spatial foraging segregation between individuals from Radio-tracking Bay versus Penguin Parade was not dependent on these 2 long foraging trips. For example, if these 2 long foraging trips were removed from the analysis, the differences in space use between sites remained (Table A2).

Median bathymetry of the Penguin Parade foraging area during incubation was significantly shallower than that of Radio-tracking Bay for both the home range and core range (Table 2). Median bathymetry of the home-range of prey encounter during guard was shallower at Penguin Parade (Table 2). No significant differences in the bathymetry between sites were found during post-guard (Table 2).

Regarding the use of the water column, prey encounter depths ranged from 2 to $35 \mathrm{~m}$ in incubation, from 2 to $61.8 \mathrm{~m}$ in guard and from 2 to $55.2 \mathrm{~m}$ in post-guard. Sex had no significant effect in the prey encounter depth for any stage (Table 3). There were no significant differences between sites in the prey encounter depth over incubation and post-guard (Table 3, Fig. 4). During the guard stage, birds from Radio-tracking Bay found prey at a significantly greater depth than those from Penguin Parade $(6 \mathrm{~m}$ deeper, LMM $\mathrm{p}<0.001$; Table 3, Fig. 4).

\section{Prey encounter rate}

The prey encounter rate on each foraging trip ranged from 10.4 to 75.0 dives $\mathrm{h}_{\mathrm{div}}{ }^{-1}$ in incubation, from 10.0 to 44.0 dives $\mathrm{h}_{\text {div }}{ }^{-1}$ in guard and from 11.0 to 32.5 dives $\mathrm{h}_{\text {div }}{ }^{-1}$ in post-guard. Sex and trip duration had no significant effect on the prey encounter rate in any stage (Table 4). At guard, birds from
Table 2. Median ( \pm median absolute deviation) bathymetry (depth in $\mathrm{m}$ ) within little penguin foraging areas of individual trips (home range $=95 \%$ kernel contour, core area $=25 \%$ kernel contour) at Penguin Parade and Radio-tracking Bay during incubation, guard and post-guard. Empirical p-value (p): the proportion of 10000 random permutations calculating a generalised Wilcoxon test between sites with a test statistic larger or equal to the observed data. Asterisks ( ${ }^{*}$ ) indicate significant differences between sites $(p<0.05)$

Table 3. Parameter estimates $(\beta)$, standard errors (SE), lower $(2.5 \%)$ and upper with a random intercept of little penguin ID and first-order autoregressive strucure for prey encounter depth. Models are fitted for incubation, guard and post'Penguin Parade'

\begin{tabular}{|lcccc|}
\hline Fixed effect & $\beta+\mathrm{SE}$ & Lower CI & Upper CI & $\mathrm{p}$ \\
\hline Incubation & & & & \\
$\quad$ Intercept & $7.6 \pm 1$ & 5.6 & 9.6 & $<0.001$ \\
Sex(male) & $-0.2 \pm 1.2$ & -2.67 & 2.21 & 0.8 \\
Site(Radio-tracking Bay) & $2.3 \pm 1.2$ & -0.17 & 4.7 & 0.07 \\
Guard & & & & \\
Intercept & $10.9 \pm 1.3$ & 8.3 & 13.5 & $<0.001$ \\
Sex(male) & $1.7 \pm 1.5$ & -1.3 & 4.8 & 0.26 \\
Site(Radio-tracking Bay) & $6 \pm 1.47$ & 2.9 & 9 & $<0.001$ \\
Post-guard & & & & \\
Intercept & $13.9 \pm 1.7$ & 10.6 & 17.2 & $<0.001$ \\
Sex(male) & $1.5 \pm 2$ & -2.9 & 5.9 & 0.48 \\
Site(Radio-tracking Bay) & $-1.3 \pm 2$ & -5.7 & 3.1 & 0.54 \\
\hline
\end{tabular}

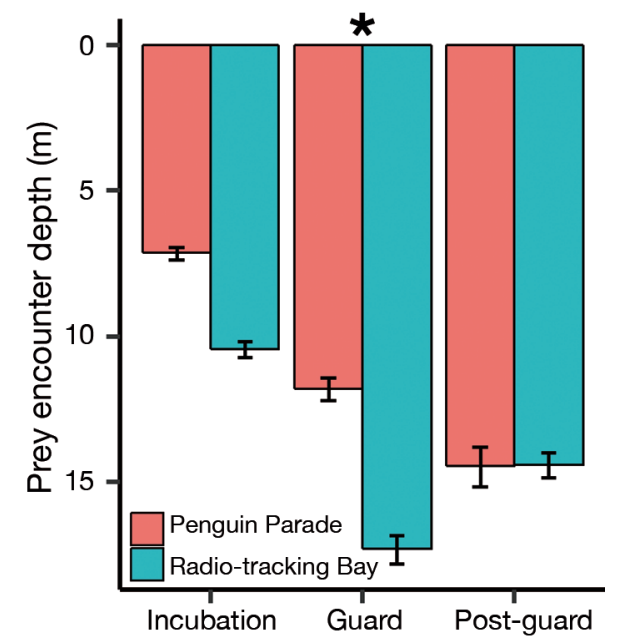

Fig. 4. Mean prey encounter depths and $95 \%$ confidence interval over the 2015/2016 breeding season of little penguins at Penguin Parade and Radio-tracking Bay. Asterisks $\left({ }^{*}\right)$ indicate significant differences between sites in guard (LMM: $\beta=6, \mathrm{SE}=1.46, t=4, \mathrm{p}<0.001$ ) 
Table 4. Parameter estimates $(\beta)$, standard errors (SE), lower $(2.5 \%)$ and upper $(97.5 \%)$ confidence intervals (CI) and p-values for the general least squared models for prey encounter rate. Models were fitted for incubation, guard and post-guard. Base level of the factor 'sex' is 'female' and base level of the factor 'site' is 'Penguin Parade'

\begin{tabular}{|lcccc|}
\hline Effect & $\beta+\mathrm{SE}$ & Lower CI & Upper CI & $\mathrm{p}$ \\
\hline Incubation & & & & \\
$\quad$ Intercept & $20.4 \pm 7.2$ & 5 & 35.9 & 0.01 \\
Sex(male) & $13.3 \pm 7.2$ & -2 & 28.7 & 0.08 \\
Site(Radio-tracking Bay) & $7.1 \pm 7.2$ & -8.3 & 22.6 & 0.34 \\
Trip duration & $2.8 \pm 2$ & -1.5 & 7 & 0.18 \\
Guard & & & & \\
Intercept & $29.5 \pm 2.2$ & 24.8 & 34.2 & $<0.001$ \\
Sex(male) & $-1.1 \pm 2.5$ & -6.4 & 4.1 & 0.6 \\
Site(Radio-tracking Bay) & $-6.1 \pm 2.5$ & -11.3 & -0.9 & 0.02 \\
Post-guard & & & & \\
Intercept & $15.7 \pm 3$ & 9.2 & 22.1 & $<0.001$ \\
Sex(male) & $2.5 \pm 3.2$ & -4.5 & 9.5 & 0.46 \\
Site(Radio-tracking Bay) & $-1.7 \pm 4$ & -10.3 & 6.8 & 0.67 \\
Trip duration & $1 \pm 1.3$ & -1.7 & 3.8 & 0.42 \\
\hline
\end{tabular}

\section{Foraging efficiency}

Over the 2 mo of the tracking period, the APMS recorded a total of 1091 foraging trips (n) with a known associated mass change for 77 different individuals (N) at Penguin Parade and 831 foraging trips for 61 different individuals at Radiotracking Bay. The body mass change per trip was significantly higher at Penguin Parade than Radio-tracking Bay in guard trips (mean $\pm \mathrm{SE}$, $302 \pm 9$ g vs. $190 \pm 17$ g; LMM p < 0.001, $\mathrm{n}=500, \mathrm{~N}=101$; Fig. 5b) and in post-guard trips $(278 \pm 7 \mathrm{~g}$ vs. $219 \pm 12$ g; LMM p < 0.001, n = 897, $\mathrm{N}=100$; Fig. 5b), but no significant differences were observed during incubation $(243 \pm 10 \mathrm{~g}$ for Penguin Parade vs. $209 \pm 12 \mathrm{~g}$ for Radiotracking Bay; LMM $\mathrm{p}=0.17, \mathrm{n}=525, \mathrm{~N}=125$; Fig. 5b). There were no significant differences between sites in adult body mass at the beginning of the trips (Table A3).
Radio-tracking Bay performed significantly fewer dives with prey encounter per hour diving than birds from Penguin Parade (6 dives fewer per hour, GLS p $<0.05$; Table 4, Fig. 5a).

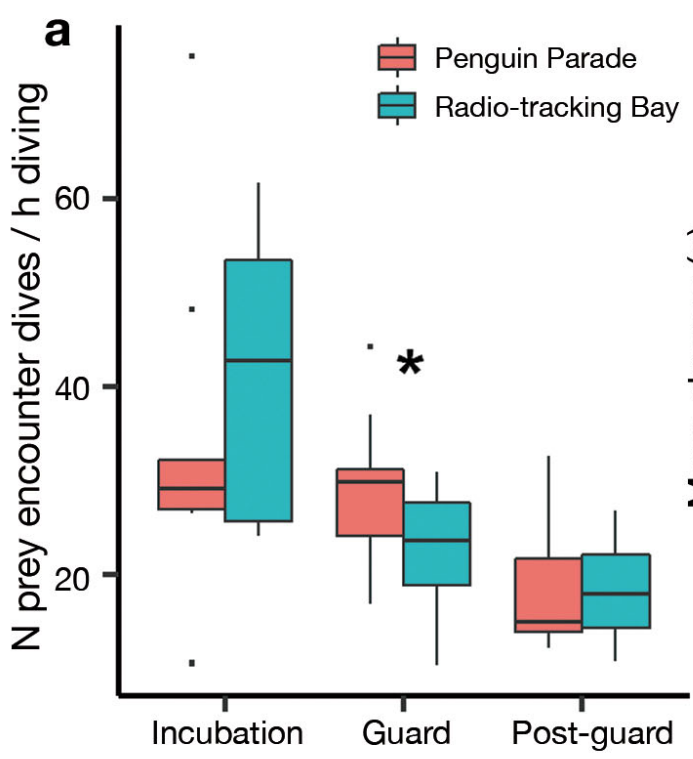

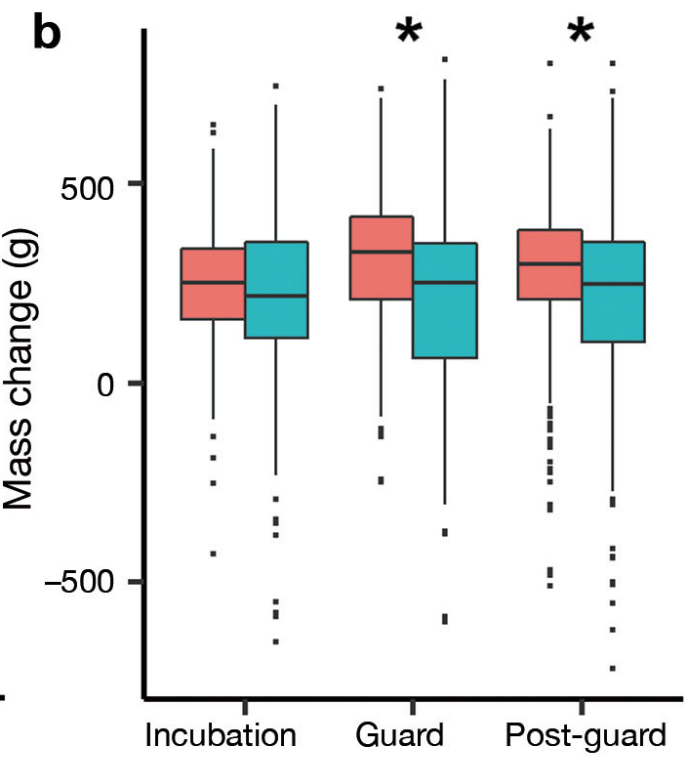

Fig. 5. (a) Prey encounter rate per breeding stage of little penguins as number of dives with prey encounter per hour diving given a foraging trip. Asteriks $\left({ }^{*}\right)$ indicate significant differences between sites in guard (GLS: $\beta=-6.1, \mathrm{SE}=2, \mathrm{p}<0.05, F$-test GLS). (b) Adult mass change per breeding stage associated with foraging trips recorded by the automated penguin monitoring systems at Penguin Parade and Radio-tracking Bay during the first clutch of the 2015/2016 breeding season. ${ }^{*}$ Significant differences in guard (LMM: $\beta=-126, \mathrm{SE}=23,95 \% \mathrm{CI}=-172,-80, t=-5.4, \mathrm{p}<0.001)$ and in post-guard between sites (LMM: $\beta=$ $-61, \mathrm{SE}=17,95 \% \mathrm{CI}=-95,-26, t=-3.5, \mathrm{p}<0.001)$. Lower and upper hinges of boxplots: first and third quartiles, respectively; bar inside box: median; whiskers: largest and smallest values $\leq 1.5 \times$ the interquartile range from the hinges; dots (outliers): data beyond the end of the whiskers 
Table 5. Number of little penguin chicks fledged per pair at Penguin Parade and Radio-tracking Bay during 2004-2015. na: data not available

\begin{tabular}{|ccc|}
\hline \multirow{2}{*}{ Year } & \multicolumn{2}{c|}{ Chicks fledged } \\
& Penguin Parade & Radio-tracking Bay \\
\hline 2015 & 2.6 & 1.7 \\
2014 & 1.5 & na \\
2013 & 0.6 & 0.7 \\
2012 & 2.1 & 1.1 \\
2011 & 1.2 & 1.0 \\
2010 & 1.7 & 1.0 \\
2009 & 1.7 & 1.2 \\
2008 & 0.6 & 1.0 \\
2007 & 1.3 & 1.0 \\
2006 & 0.9 & 0.8 \\
2005 & 1.3 & 1.1 \\
2004 & 0.8 & 0.5 \\
\hline
\end{tabular}

\section{Reproductive performance}

Breeding success was consistently 10-30\% higher at Penguin Parade than Radio-tracking Bay from 2004/2005 until 2015/2016 (Table 5). The breeding success in 2015/2016 was 2.6 chicks fledged per pair at Penguin Parade and 1.7 chicks fledged per pair at Radio-tracking Bay (Table 5). There were no significant differences in the peak chick mass between sites $(1180 \pm 9$ vs. $1173 \pm 11$ g; LM: $\beta=-13.59, \mathrm{SE}=$ $15, t=-0.87, \mathrm{p}=0.4)$.

\section{DISCUSSION}

Using GPS, depth and accelerometer data loggers simultaneously, we observed a strong spatial segregation between 2 little penguin breeding sites that are only $2 \mathrm{~km}$ apart, although their home ranges have a high potential to overlap given that the species' foraging range is $20 \mathrm{~km}$ during the breeding season (Collins et al. 1999). Birds preferred areas adjacent to their own breeding site in accordance with Cairns (1989) and Wakefield et al. (2013), suggesting that foraging areas are site-specific within the colony. Several seabirds have shown partial or complete horizontal spatial segregation among colonies (e.g. Grémillet et al. 2004, Wakefield et al. 2013), even at small scale (Masello et al. 2010, Ceia et al. 2015). Other studies, for example, have used 3dimensional foraging behaviour to test for competition between congeneric penguins (Cimino et al. 2016) and to quantify foraging volumes (Ainley et al. 2015). Here, we used acceleration data loggers in addition to GPS and depth data considered by the aforementioned studies. This approach allowed us to not only identify horizontal segregation between the site-specific foraging areas, but also vertical differences between sites in the spatial distribution of prey encounters over the breeding season, linking birds' movements with their behaviour and prey distribution in 3 dimensions.

Birds from Penguin Parade and Radio-tracking Bay travelled in different directions relative to their breeding sites, leading to a significant within-colony spatial foraging segregation between sites. Centralplace foragers such as little penguins can initiate their foraging trips on a bearing consistent with the general orientation of their breeding site (Robson et al. 2004) and return to previous successful foraging grounds (Carroll et al. 2018). Further, foraging aggregations of little penguins have been associated with the departure time from the colony (Sutton et al. 2017). In particular, birds from Phillip Island are known to form non-random groups when departing (Daniel et al. 2007), providing the opportunity for foraging aggregations to form. Thus, the use of site-specific pathways may facilitate information exchange among conspecifics from the same breeding site and their aggregation at sea, mediating the foraging segregation observed here.

The spatial segregation between sites intensified throughout the breeding season, and prey encounter depth increased from incubation to chick-rearing. Chick-rearing is a highly demanding period due to the increasing energetic requirements of the young combined with the central-place foraging constraints (Gales \& Green 1990, Bevan et al. 2002). Thus, indirect competition for food resources is expected to increase from incubation to chick-rearing, and parents can meet this high energetic demand by increasing foraging effort, either by diving deeper (e.g. gentoo penguins Pygoscelis papua, Ratcliffe et al. 2018; northern rockhopper penguins Eudyptes moseleyi, Booth et al. 2018), switching diet to more energetic prey (e.g. gentoo penguins, Handley et al. 2017) or expanding the foraging area (e.g. Adélie penguins $P$. adeliae, Ainley et al. 2004). Expanding the foraging area during the guard stage compared to incubation is not an option for little penguins, and at-sea density of penguins is expected to be higher, as guard trips are always 1 d long (Chiaradia \& Kerry 1999, Chiaradia \& Nisbet 2006), whereas incubation trips can last several days (Kato et al. 2008). Instead, birds seemed to mitigate within-site competition by diving deeper (i.e. deeper prey encounters) and between-site competition by increasing segregation level. Wakefield et al. (2013) were only able to reproduce the spatial 
segregation between northern gannet Morus bassanus colonies if models incorporated density-dependence constraints (Ashmole's halo), interactions with neighbouring colonies (hinterland model), information transfer and memory. Our results suggest similar underpinning mechanisms for the within-colony spatial segregation observed here.

Inter- and intra-specific competition and changes in environmental conditions have the potential to reduce the abundance and availability of prey and enlarge the foraging area (Lewis et al. 2001, Pichegru et al. 2013). Areas off Phillip Island support a high biomass and diversity of seabirds (Norman 1991, Dann et al. 2003) and a large Australian fur seal Arctocephalus pusillus doriferus colony (Kirkwood et al. 2005). Little penguins in our study area are at the top of the food chain (Chiaradia et al. 2016). Small pelagic fish are their key prey, and other common prey include barracouta Thyrsites atun, red cod Pseudophysis bachus and arrow squid Nototodarus gouldi (Chiaradia et al. 2003, 2010). These are also significant prey for other predators within the area, such as short-tailed shearwaters Puffinus tenuirostris (Montague et al. 1986), crested terns Thalasseus bergii (Chiaradia et al. 2002) and Australian fur seals (Deagle et al. 2009). Little penguins may compete with crested terns for food resources or be affected by the similar prey availability variation, as low numbers of breeding crested terns have coincided with low breeding success of little penguins in the past (Chiaradia et al. 2002). However, knowledge is scarce about inter-specific competition interactions among marine predators off Phillip Island and how they might affect penguins' foraging behaviour.

Bathymetry is another important factor influencing prey availability and at-sea distribution of many marine predators, including marine mammals and seabirds (e.g. Guinet et al. 2001, Yen et al. 2004, Weimerskirch et al. 2007). Colonies with shallower waters are favourable to little penguins, as birds show a lower diving effort (i.e. total diving duration per hour) compared to conspecifics from colonies with deeper waters (Chiaradia et al. 2007, Meyer et al. 2017). Here, the kernel UDs of prey encounters showed that the Radio-tracking Bay birds used a deeper and larger foraging area than birds from Penguin Parade throughout the breeding season. Therefore, individuals from Radio-tracking Bay were not only exposed to less favourable bathymetry conditions, but might have also experienced higher levels of intra-specific competition within their area, reducing prey availability in the site vicinity, which forced birds to forage further in accordance with Ashmole's halo theory
(Ashmole 1963). Deeper prey encounters in the water column could be a mechanism by birds from Radiotracking Bay to expand their niche width and reduce within-site competition, as has been observed in gentoo penguins (Ratcliffe et al. 2018). At the same time, generalist marine predators, such as little penguins (Chiaradia et al. 2010), modify their foraging behaviour according to prey type (Elliott et al. 2008) and match the 3-dimensional distribution of their prey (Carroll et al. 2017). Hence, differences in prey encounter depths could also be a response to availability of different prey types at each site or different prey vertical distribution. However, diet composition in the year of this study did not differ between sites, with birds foraging mainly on sardines (C. Cavallo unpubl. data). Therefore, rather than differences in diet, we suggest that bathymetry and higher competition within Radio-tracking Bay foraging grounds could explain the higher diving effort (i.e. deeper prey encounters) and the lower prey encounter rate at this site during chick-rearing, leading to a lower foraging efficiency (i.e. lower mass gain after foraging trips).

Seabirds with a small foraging range are highly sensitive to variations in local food availability and abundance (Chiaradia et al. 2010, Kowalczyk et al. $2014,2015)$. All of the foraging parameters analysed here (i.e. size of foraging area, prey encounter depth, prey encounter rate and body mass gain) suggest that Penguin Parade birds foraged more efficiently in more profitable foraging grounds. Higher body mass gain after foraging trips mean larger chick meals (Saraux et al. 2011, 2016). From albatrosses to little penguins, when chicks receive large and frequent meals they have a higher probability of fledging (Huin et al. 2000, Saraux et al. 2016). Hence, the higher body mass gain by Penguin Parade adults during the 2015/2016 season could result in larger meals for their chicks and a higher breeding success. The breeding records for the last $12 \mathrm{yr}$ indicated that Penguin Parade has consistently been more productive than Radio-tracking Bay, suggesting that this site is overall more successful. Nonetheless, both sites were highly productive $\left(>1.5\right.$ chicks fledged pair $\left.{ }^{-1}\right)$ in the 2015/2016 season, which is indicative of no food limitations (Chiaradia \& Nisbet 2006), and raised chicks with similar peak chick mass. These findings suggest that when food resources are not limiting, the different foraging strategies from each site and their costs result in chicks with similar condition, and other factors, such as nest predation (Ekanayake et al. 2015), might be partially responsible for the differences in breeding success. Hence, the link between foraging behaviour and breeding success remains 
unknown. Yet, birds from Penguin Parade could have an advantage by foraging more efficiently and in more profitable areas than those from Radio-tracking Bay. Future studies should investigate how the differences in foraging efficiency between sites influence chick fitness in years of food shortage. Overall, our findings show that differences in foraging behaviour of birds from the same colony were associated with breeding sites, and the site with the highest foraging efficiency produced more fledglings.

\section{CONCLUSIONS}

Bio-logging data have been used worldwide in numerous studies to identify key areas for marine megafauna (e.g. Bailey \& Thompson 2009, Lascelles et al. 2016, Augé et al. 2018), and seabird biodiversity hotspots in the oceans coincide with areas in serious need of conservation (Ramírez et al. 2017). However, incorporation of these data into conservation strategies remains underutilised (Hays et al. 2016). Our findings highlight the importance of understanding fine-scale spatial segregation and intra-specific competition mechanisms within large colonies and their consequences for seabird foraging and breeding ecology. Within-colony segregation may be the norm when large seabird colonies are spatially structured in breeding sites, leading to site-specific foraging behaviours. Therefore, we argue that assessing more than 1 breeding site, over different breeding stages, is necessary to capture the foraging behaviour and breeding success variability within a colony, providing meaningful knowledge on marine spatial usage to obtain accurate population assessments and to incorporate into conservation strategies.

Acknowledgements. We thank Phillip Island Nature Parks for their support to conduct the fieldwork, in particular P. Wasiak and L. Renwick for providing field training. We also thank R. Holmberg for assistance with the automated penguin monitoring system data processing and all volunteers for help during fieldwork. We thank the IPHC-DEPE for providing expertise on the GPS customisation. S.S. was supported by an Australian Government Research Training Program (RTP) Scholarship and Monash University. Project funding was provided by the Australian Research Council (Linkage Project LP140100404), Monash University, Phillip Island Nature Parks and Institut Pluridisciplinaire Hubert Curien.

\section{LITERATURE CITED}

Ainley DG, Ford RG, Brown ED, Suryan RM, Irons DB (2003) Prey resources, competition, and geographic structure of kittiwake colonies in Prince William Sound. Ecology 84: 709-723
Ainley DG, Ribic CA, Ballard G, Heath S and others (2004) Geographic structure of Adélie penguin populations: overlap in colony-specific foraging areas. Ecol Monogr 74:159-178

Ainley DG, Ballard G, Jones RM, Jongsomjit D, Pierce SD, Smith WO Jr, Veloz S (2015) Trophic cascades in the western Ross Sea, Antarctica: revisited. Mar Ecol Prog Ser 534:1-16

Arnould JPY, Dann P, Cullen JM (2004) Determining the sex of little penguins Eudyptula minor in northern Bass Strait using morphometric measurements. Emu 104:261-265

Ashmole NP (1963) The regulation of numbers of tropical oceanic birds. Ibis 103:458-473

Augé AA, Dias MP, Lascelles B, Baylis AMM and others (2018) Framework for mapping key areas for marine megafauna to inform marine spatial planning: the Falkland Islands case study. Mar Policy 92:61-72

Bailey H, Thompson PM (2009) Using marine mammal habitat modelling to identify priority conservation zones within a marine protected area. Mar Ecol Prog Ser 378: 279-287

* Bevan R, Butler P, Woakes A, Boyd I (2002) The energetics of gentoo penguins, Pygoscelis papua, during the breeding season. Funct Ecol 16:175-190

*Booth JM, McQuaid CD (2013) Northern rockhopper penguins prioritise future reproduction over chick provisioning. Mar Ecol Prog Ser 486:289-304

Booth JM, Steinfurth A, Fusi M, Cuthbert RJ, McQuaid CD (2018) Foraging plasticity of breeding northern rockhopper penguins, Eudyptes moseleyi, in response to changing energy requirements. Polar Biol 41:1815-1826

*Beed GA, Bowen WD, McMillan JI, Leonard ML (2006) Sexual segregation of seasonal foraging habitats in a nonmigratory marine mammal. Proc R Soc B 273:2319-2326

* Cairns DK (1989) The regulation of seabird colony size: a hinterland model. Am Nat 134:141-146

KCalenge C (2006) The package 'adehabitat' for the R software: a tool for the analysis of space and habitat use by animals. Ecol Model 197:516-519

Carroll G, Cox M, Harcourt R, Pitcher BJ, Slip D, Jonsen I (2017) Hierarchical influences of prey distribution on patterns of prey capture by a marine predator. Funct Ecol 31:1750-1760

Carroll G, Harcourt R, Pitcher BJ, Slip D, Jonsen I (2018) Recent prey capture experience and dynamic habitat quality mediate short-term foraging site fidelity in a seabird. Proc R Soc B 285:20180788

Ceia FR, Paiva VH, Ceia RS, Hervías S, Garthe S, Marques JC, Ramos JA (2015) Spatial foraging segregation by close neighbours in a wide-ranging seabird. Oecologia 177:431-440

Chiaradia AF, Kerry KR (1999) Daily nest attendance and breeding performance in the little penguin Eudyptula minor at Phillip Island, Australia. Mar Ornithol 27:13-20

Chiaradia A, Nisbet IC (2006) Plasticity in parental provisioning and chick growth in little penguins Eudyptula minor in years of high and low breeding success. Ardea 94:257-270

Chiaradia A, Dann P, Jessop R, Collins P (2002) The diet of crested tern (Sterna bergii) chicks on Phillip Island, Victoria, Australia. Emu 102:367-371

Chiaradia A, Costalunga A, Kerry K (2003) The diet of little penguins (Eudyptula minor) at Phillip Island, Victoria, in the absence of a major prey-pilchard (Sardinops sagax). Emu 103:43-48 
Chiaradia A, Ropert-Coudert Y, Kato A, Mattern T, Yorke J (2007) Diving behaviour of little penguins from four colonies across their whole distribution range: bathymetry affecting diving effort and fledging success. Mar Biol 151:1535-1542

Chiaradia A, Forero MG, Hobson KA, Cullen JM (2010) Changes in diet and trophic position of a top predator 10 years after a mass mortality of a key prey. ICES J Mar Sci 67:1710-1720

Chiaradia A, Ramírez F, Forero MG, Hobson KA (2016) Stable isotopes $\left(\delta^{13} \mathrm{C}, \delta^{15} \mathrm{~N}\right)$ combined with conventional dietary approaches reveal plasticity in central-place foraging behavior of little penguins Eudyptula minor. Front Ecol Evol 3:154

Cimino MA, Moline MA, Fraser WR, Patterson-Fraser DL, Oliver MJ (2016) Climate-driven sympatry may not lead to foraging competition between congeneric top-predators. Sci Rep 6:18820

Cleasby IR, Wakefield ED, Bodey TW, Davies RD and others (2015) Sexual segregation in a wide-ranging marine predator is a consequence of habitat selection. Mar Ecol Prog Ser 518:1-12

* Collins M, Cullen JM, Dann P (1999) Seasonal and annual foraging movements of little penguins from Phillip Island, Victoria. Wildl Res 26:705-721

Dall SR, Giraldeau LA, Olsson O, McNamara JM, Stephens DW (2005) Information and its use by animals in evolutionary ecology. Trends Ecol Evol 20:187-193

* Danchin E, Wagner RH (1997) The evolution of coloniality: the emergence of new perspectives. Trends Ecol Evol 12: 342-347

* Daniel TA, Chiaradia A, Logan M, Quinn GP, Reina RD (2007) Synchronized group association in little penguins, Eudyptula minor. Anim Behav 74:1241-1248

WDann P (1991) Distribution, population trends and factors influencing the population size of little penguins Eudyptula minor on Phillip Island, Victoria. Emu 91:263-272

Dann P, Arnould JP, Jessop R, Healy M (2003) Distribution and abundance of seabirds in Western Port, Victoria. Emu 103:307-313

Deagle BE, Kirkwood R, Jarman SN (2009) Analysis of Australian fur seal diet by pyrosequencing prey DNA in faeces. Mol Ecol 18:2022-2038

Dubois F, Cézilly F, Pagel M (1998) Mate fidelity and coloniality in waterbirds: a comparative analysis. Oecologia 116:433-440

Ekanayake KB, Sutherland DR, Dann P, Weston MA (2015) Out of sight but not out of mind: corvids prey extensively on eggs of burrow-nesting penguins. Wildl Res 42: 509-517

* Elliott KH, Woo K, Gaston AJ, Benvenuti S, Dall'Antonia L, Davoren GK (2008) Seabird foraging behaviour indicates prey type. Mar Ecol Prog Ser 354:289-303

Elliott KH, Woo KJ, Gaston AJ, Benvenuti S, Dall'Antonia L, Davoren GK (2009) Central-place foraging in an arctic seabird provides evidence for Storer-Ashmole's halo. Auk 126:613-625

Evans JC, Votier SC, Dall SR (2016) Information use in colonial living. Biol Rev Camb Philos Soc 91:658-672

Fieberg J, Kochanny CO (2005) Quantifying home-range overlap: the importance of the utilization distribution. J Wildl Manag 69:1346-1359

Forero M, Tella J, Hobson K, Bertellotti M, Blanco G (2002) Conspecific food competition explains variability in colony size: a test in Magellanic penguins. Ecology 83:3466-3475
Furness R, Birkhead T (1984) Seabird colony distributions suggest competition for food supplies during the breeding season. Nature 311:655-656

Gales R, Green B (1990) The annual energetics cycle of little penguins (Eudyptula minor). Ecology 71:2297-2312

Gleiss AC, Wilson RP, Shepard ELC (2011) Making overall dynamic body acceleration work: on the theory of acceleration as a proxy for energy expenditure. Methods Ecol Evol 2:23-33

Grémillet D, Dell'Omo G, Ryan PG, Peters G, RopertCoudert Y, Weeks SJ (2004) Offshore diplomacy, or how seabirds mitigate intra-specific competition: a case study based on GPS tracking of Cape gannets from neighbouring colonies. Mar Ecol Prog Ser 268:265-279

Guinet C, Dubroca L, Lea MA, Goldsworthy S and others (2001) Spatial distribution of foraging in female Antarctic fur seals Arctocephalus gazella in relation to oceanographic variables: a scale-dependent approach using geographic information systems. Mar Ecol Prog Ser 219: 251-264

*Handley JM, Connan M, Baylis AM, Brickle P, Pistorius P (2017) Jack of all prey, master of some: influence of habitat on the feeding ecology of a diving marine predator. Mar Biol 164:82

*Hays GC, Ferreira LC, Sequeira AMM, Meekan MG and others (2016) Key questions in marine megafauna movement ecology. Trends Ecol Evol 31:463-475

Hijmans RJ (2016) geosphere: spherical trigonometry. R package version 1.5-5. https://cran.r-project.org/src/contrib/ Archive/geosphere/geosphere_1.5-5.tar.gz

*Hipfner JM, Charette MR, Blackburn GS (2007) Subcolony variation in breeding success in the tufted puffin (Fratercula cirrhata): association with foraging ecology and implications. Auk 124:1149-1157

*Huin N, Prince PA, Briggs DR (2000) Chick provisioning rates and growth in black-browed albatross Diomedea melanophris and grey-headed albatross D. chrysostoma at Bird Island, South Georgia. Ibis 142:550-565

Jovani R, Mavor R, Oro D (2008) Hidden patterns of colony size variation in seabirds: a logarithmic point of view. Oikos 117:1774-1781

KKato A, Ropert-Coudert Y, Grémillet D, Cannell B (2006) Locomotion and foraging strategy in foot-propelled and wing-propelled shallow-diving seabirds. Mar Ecol Prog Ser 308:293-301

Kato A, Ropert-Coudert Y, Chiaradia A (2008) Regulation of trip duration by an inshore forager, the little penguin (Eudyptula minor), during incubation. Auk 125:588-593

Kerry K, Clarke J, Else G (1993) The use of an automated weighing and recording system for the study of the biology of Adélie penguins (Pygoscelis adeliae). Proc NIPR Symp Polar Biol 6:62-75

Kirkwood R, Gales R, Terauds A, Arnould JPY and others (2005) Pup production and population trends of the Australian fur seal (Arctocephalus pusillus doriferus). Mar Mamm Sci 21:260-282

Kowalczyk ND, Chiaradia A, Preston TJ, Reina RD (2014) Linking dietary shifts and reproductive failure in seabirds: a stable isotope approach. Funct Ecol 28:755-765

Kowalczyk ND, Chiaradia A, Preston TJ, Reina RD (2015) Fine-scale dietary changes between the breeding and non-breeding diet of a resident seabird. R Soc Open Sci 2:140291

K Lascelles BG, Taylor PR, Miller MGR, Dias MP and others (2016) Applying global criteria to tracking data to define 
important areas for marine conservation. Divers Distrib 22:422-431

Lewis S, Sherratt T, Hamer K, Wanless S (2001) Evidence of intra-specific competition for food in a pelagic seabird. Nature 412:816-819

Masello JF, Mundry R, Poisbleau M, Demongin L, Voigt CC, Wikelski M, Quillfeldt P (2010) Diving seabirds share foraging space and time within and among species. Ecosphere 1:art19

McInnes AM, McGeorge C, Ginsberg S, Pichegru L, Pistorius PA (2017) Group foraging increases foraging efficiency in a piscivorous diver, the African penguin. R Soc Open Sci 4:170918

* Meyer X, MacIntosh AJ, Chiaradia A, Kato A, Mattern T, Sueur C, Ropert-Coudert Y (2017) Shallow divers, deep waters and the rise of behavioural stochasticity. Mar Biol 164:149

Møller AP (1987) Advantages and disadvantages of coloniality in the swallow, Hirundo rustica. Anim Behav 35: 819-832

Montague TL, Cullen JM, Fitzherbert K (1986) The diet of the short-tailed shearwater Puffinus tenuirostris during its breeding season. Emu 86:207-213

Neubert K, Brunner E (2007) A studentized permutation test for the non-parametric Behrens-Fisher problem. Comput Stat Data Anal 51:5192-5204

Norman FI (1991) Distribution and abundance of seabirds off Phillip Island and within Port Phillip Bay, Victoria, 1986-1988. Emu 91:377-394

North BV, Curtis D, Sham PC (2002) A note on the calculation of empirical $\mathrm{p}$ values from Monte Carlo procedures. Am J Hum Genet 71:439-441

Pelletier L, Chiaradia A, Kato A, Ropert-Coudert Y (2014) Fine-scale spatial age segregation in the limited foraging area of an inshore seabird species, the little penguin. Oecologia 176:399-408

* Pichegru L, Cook T, Handley J, Voogt N, Watermeyer J, Nupen L, McQuaid CD (2013) Sex-specific foraging behaviour and a field sexing technique for Endangered African penguins. Endang Species Res 19:255-264

Pinheiro J, Bates D, DebRoy S, Sarkar D, R Core Team (2016) nlme: linear and nonlinear mixed effects models. R package version 3.1-125. https://cran.r-project.org/src/ contrib/Archive/nlme/nlme_3.1-125.tar.gz

* Preston TJ, Ropert-Coudert Y, Kato A, Chiaradia A, Kirkwood R, Dann P, Reina RD (2008) Foraging behaviour of little penguins Eudyptula minor in an artificially modified environment. Endang Species Res 4:95-103

Preston TJ, Chiaradia A, Caarels SA, Reina RD (2010) Fine scale biologging of an inshore marine animal. J Exp Mar Biol Ecol 390:196-202

R Core Team (2017) R: a language and environment for statistical computing. R Foundation for Statistical Computing, Vienna

* Ramírez F, Afán I, Davis LS, Chiaradia A (2017) Climate impacts on global hot spots of marine biodiversity. Sci Adv 3:e1601198

Ramos JA, Monteiro LR, Sola E, Moniz Z (1997) Characteristics and competition for nest cavities in burrowing Procellariiformes. Condor 99:634-641

Ratcliffe N, Adlard S, Stowasser G, McGill R (2018) Dietary divergence is associated with increased intra-specific competition in a marine predator. Sci Rep 8:6827

Robinson SK (1985) Coloniality in the yellow-rumped cacique as a defense against nest predators. Auk 102:506-519
Robinson EJ, Richardson TO, Sendova-Franks AB, Feinerman O, Franks NR (2009) Radio tagging reveals the roles of corpulence, experience and social information in ant decision making. Behav Ecol Sociobiol 63:627-636

Robson BW, Goebel ME, Baker JD, Ream RR and others (2004) Separation of foraging habitat among breeding sites of a colonial marine predator, the northern fur seal (Callorhinus ursinus). Can J Zool 82:20-29

* Rolland C, Danchin E, de Fraipont M (1998) The evolution of coloniality in birds in relation to food, habitat, predation, and life-history traits: a comparative analysis. Am Nat 151:514-529

Ropert-Coudert Y, Wilson RP, Daunt F, Kato A (2004) Patterns of energy acquisition by a central place forager: benefits of alternating short and long foraging trips. Behav Ecol 15:824-830

* Ropert-Coudert Y, Kato A, Wilson RP, Cannell B (2006) Foraging strategies and prey encounter rate of free-ranging little penguins. Mar Biol 149:139-148

* Ropert-Coudert Y, Knott N, Chiaradia A, Kato A (2007) How do different data logger sizes and attachment positions affect the diving behaviour of little penguins? Deep Sea Res II 54:415-423

Ruxton GD, Neuhäuser M (2013) Improving the reporting of $\mathrm{p}$ values generated by randomization methods. Methods Ecol Evol 4:1033-1036

Saraux C, Robinson-Laverick SM, Le Maho Y, Ropert-Coudert Y, Chiaradia A (2011) Plasticity in foraging strategies of inshore birds: how little penguins maintain body reserves while feeding offspring. Ecology 92:1909-1916

Saraux C, Chiaradia A, Salton M, Dann P, Viblanc VA (2016) Negative effects of wind speed on individual foraging performance and breeding success in little penguins. Ecol Monogr 86:61-77

* Shepard ELC, Wilson RP, Quintana F, Gómez Laich A and others (2008) Identification of animal movement patterns using tri-axial accelerometry. Endang Species Res 10: 47-60

Storer RW (1952) A comparison of variation, behavior and evolution in the sea bird genera Uria and Cepphus. Univ Calif Publ Zool 51:121-122

K Sutherland D, Dann P (2014) Population trends in a substantial colony of little penguins: three independent measures over three decades. Biodivers Conserv 23:241-250

* Sutton GJ, Hoskins AJ, Berlincourt M, Arnould JPY (2017) Departure time influences foraging associations in little penguins. PLOS ONE 12:e0182734

*Waggitt JJ, Briffa M, Grecian WJ, Newton J, Patrick SC, Stauss C, Votier SC (2014) Testing for sub-colony variation in seabird foraging behaviour: ecological and methodological consequences for understanding colonial living. Mar Ecol Prog Ser 498:275-285

*Wakefield ED, Bodey TW, Bearhop S, Blackburn J and others (2013) Space partitioning without territoriality in gannets. Science 341:68-70

*Watanuki Y, Wanless S, Harris M, Lovvorn JR, Miyazaki M, Tanaka H, Sato K (2006) Swim speeds and stroke patterns in wingpropelled divers: a comparison among alcids and a penguin. J Exp Biol 209:1217-1230

* Weimerskirch H, Cherel Y, Cuenot-Chaillet F, Ridoux V (1997) Alternative foraging strategies and resource allocation by male and female wandering albatrosses. Ecology 78:2051-2063

*Weimerskirch H, Le Corre M, Ropert-Coudert Y, Kato A, Marsac F (2006) Sex-specific foraging behaviour in a 
seabird with reversed sexual dimorphism: the red-footed booby. Oecologia 146:681-691

Weimerskirch H, Pinaud D, Pawlowski F, Bost CA (2007) Does prey capture induce area-restricted search? A finescale study using GPS in a marine predator, the wandering albatross. Am Nat 170:734-743

Weimerskirch H, Bertrand S, Silva J, Marques JC, Goya E (2010) Use of social information in seabirds: compass rafts indicate the heading of food patches. PLOS ONE 5: e9928

Wittenberger JF, Hunt GL (1985) The adaptive significance of coloniality in birds. In: Farner DS, King JR, Parkes KC (eds) Avian biology VIII. Academic Press, Orlando, FL, p 1-78

Yen PP, Sydeman WJ, Hyrenbach KD (2004) Marine bird and cetacean associations with bathymetric habitats and shallow-water topographies: implications for trophic transfer and conservation. J Mar Syst 50:79-99

Zimmer I, Ropert-Coudert Y, Kato A, Ancel A, Chiaradia A (2011a) Does foraging performance change with age in female little penguins (Eudyptula minor)? PLOS ONE 6: e16098

Zimmer I, Ropert-Coudert Y, Poulin N, Kato A, Chiaradia A (2011b) Evaluating the relative importance of intrinsic and extrinsic factors on the foraging activity of top predators: a case study on female little penguins. Mar Biol 158:715-722

Zuur A, Ieno EN, Walker N, Saveliev AA, Smith GM (2009) Mixed effects models and extensions in ecology with $\mathrm{R}$. In: Dietz K, Gail M, Samet JM, Singer B, Tsiatis A (eds) Statistics for biology and health. Springer, New York, NY, p 101-142

\section{Appendix}

Table A1. Total areas $\left(\mathrm{km}^{2}\right)$ of kernel utilisation distribution (UD) for the locations of prey encounters at Penguin Parade and Radio-tracking Bay during the 2015/2016 little penguin breeding season

\begin{tabular}{|lccc|}
\hline Stage & $\%$ UD & $\begin{array}{c}\text { Penguin } \\
\text { Parade }\end{array}$ & $\begin{array}{c}\text { Radio-tracking } \\
\text { Bay }\end{array}$ \\
\hline Incubation & 95 & 1038 & 4099 \\
& 50 & 296 & 519 \\
Guard & 25 & 122 & 152 \\
& 95 & 438 & 716 \\
Post-guard & 50 & 119 & 205 \\
& 25 & 53 & 84 \\
& 95 & 442 & 4562 \\
& 50 & 109 & 1004 \\
& 25 & 45 & 289 \\
\hline
\end{tabular}

Table A2. Estimated utilisation distribution (UD) overlap index (UDOI) in UDs between Penguin Parade and Radiotracking Bay little penguins within incubation and postguard breeding stages after dropping the long trips. Empirical p-value (p): the proportion of 1000 randomised overlaps that were smaller than the observed overlap. Randomised overlaps were only calculated if observed overlap was $>0$

\begin{tabular}{|lccc|}
\hline \multirow{2}{*}{ UD $(\%)$} & Stage & UDOI & $p$ \\
\hline 95 & Incubation & 0.45 & 0.037 \\
& Post-guard & 0.11 & 0.001 \\
50 & Incubation & 0.003 & 0.001 \\
& Post-guard & 0 & - \\
25 & Incubation & 0 & - \\
& Post-guard & 0 & - \\
\hline
\end{tabular}

Table A3. Parameter estimates $(\beta)$, standard errors (SE), lower $(2.5 \%)$ and upper $(97.5 \%)$ confidence intervals (CI) and p-values for the linear mixed effect models with a random intercept of bird ID for little penguin adult body mass at the beginning of foraging trips. Models were fitted for incubation, guard and postguard. Base level of the factor 'site' is 'Penguin Parade'

\begin{tabular}{|lcccc|}
\hline Fixed effect & $\beta+\mathrm{SE}$ & Lower CI & Upper CI & $\mathrm{p}$ \\
\hline Incubation & & & & \\
$\quad$ Intercept & $1097 \pm 13.5$ & 1071 & 1124 & $<0.001$ \\
$\quad$ Site(Radio-tracking Bay) & $-24.7 \pm 19$ & -62 & 13 & 0.2 \\
Guard & & & & \\
$\quad$ Intercept & $1067 \pm 12$ & 1044 & 1091 & $<0.001$ \\
$\quad$ Site(Radio-tracking Bay) & $-30.8 \pm 20$ & -71 & 9 & 0.13 \\
Post-guard & & & & \\
Intercept & $1075 \pm 11$ & 1055 & 1096 & $<0.001$ \\
$\quad$ Site(Radio-tracking Bay) & $-20 \pm 18$ & -55 & 14 & 0.25 \\
\hline
\end{tabular}

Editorial responsibility: Kyle Elliott, Sainte-Anne-de-Bellevue, Québec, Canada
Submitted: June 21, 2018; Accepted: September 26, 2018 Proofs received from author(s): October 25, 2018 